\title{
Optimum Sample Thickness for Trace Analyte Detection with Field- Resolved Infrared Spectroscopy
}

\author{
Marinus Huber,* Michael Trubetskov, Syed A. Hussain, Wolfgang Schweinberger, Christina Hofer, \\ and Ioachim Pupeza*
}

Cite This: Anal. Chem. 2020, 92, 7508-7514

Read Online

\section{ACCESS | Lill Metrics \& More | 回 Article Recommendations | (1) Supporting Information}

ABSTRACT: The strong absorption of liquid water in the infrared (IR) molecular fingerprint region constitutes a challenge for applications of vibrational spectroscopy in chemistry, biology, and medicine. While high-power IR laser sources enable the penetration of ever thicker aqueous samples, thereby mitigating the detrimental effects of strong attenuation on detection sensitivity, a basic advantage of heterodyne-measurement-based methods hasto the best of our knowledge-not been harnessed in broadband IR measurements to date. Here, employing field-resolved spectroscopy (FRS), we demonstrate in theory and experiment fundamental advantages of techniques whose signal-to-noise ratio (SNR) scales linearly with the electric field over those whose SNR

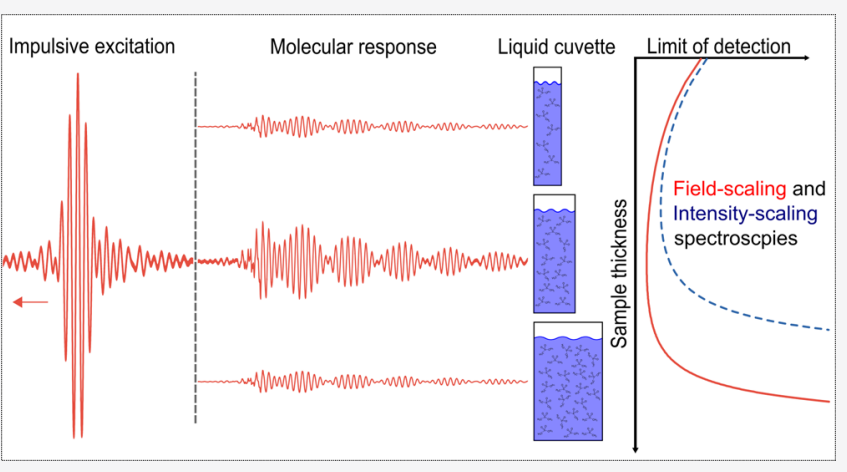
scales linearly with radiation intensity, including conventional Fourier-transform infrared (FTIR) and direct absorption spectroscopy. Field-scaling brings about two major improvements. First, it squares the measurement dynamic range. Second, we show that the optimum interaction length with samples for SNR-maximized measurements is twice the value usually considered to be optimum for FTIR devices. In order to take full advantage of these properties, the measurement must not be significantly affected by technical noise, such as intensity fluctuations, which are common for high-power sources. Recently, it has been shown that subcycle, nonlinear gating of the molecular fingerprint signal renders FRS robust against intensity noise. Here, we quantitatively demonstrate this advantage of FRS for thick aqueous samples. We report sub$\mu \mathrm{g} / \mathrm{mL}$ detection sensitivities for transmission path lengths up to $80 \mu \mathrm{m}$ and a limit of detection in the lower $\mu \mathrm{g} / \mathrm{mL}$ range for transmission paths as long as $200 \mu \mathrm{m}$.

$\mathrm{M}$ olecular vibrational spectroscopies, such as Raman and infrared (IR) spectroscopies, are versatile tools for delivering chemical and molecular information on complex samples in a fast, reliable, and label-free manner. ${ }^{1}$ Every molecule exhibits a unique spectrum of vibrational eigenstates, $^{2}$ leading to highly specific signals when a molecular ensemble (for instance biofluids or tissue) is subjected to spectroscopic interrogation. This "spectral fingerprint" contains a wealth of information about the molecular composition, structure, and conformation. ${ }^{1,2}$ This underlies numerous applications in biology and medicine, such as comprehending protein dynamics and folding, ${ }^{3}$ identifying cell phenotypes, ${ }^{4}$ quantifying blood-based clinical parameters, ${ }^{5}$ and detecting cancerous states in tissues ${ }^{6}$ and biofluids. ${ }^{7,8}$

Typically, the study of biological systems is preferably performed in their natural aqueous environment. While Raman spectroscopy is well suited for transmission measurements thereof, it suffers from weak signals. ${ }^{1}$ Broadband IR spectroscopy, on the other hand, profits from large interaction cross sections, potentially affording a unique combination of detection sensitivity and molecular coverage. ${ }^{1}$ However, the strong absorbance of (liquid) water in this spectral range has severely limited the applicability of IR vibrational spectroscopy (and microscopy) in transmission geometry so far. In fact, in table-top Fourier-transform IR (FTIR) spectrometry setups, the most commonly employed technology, the transmission path length needs to be limited to $<10 \mu \mathrm{m}$ due to the modest brightness of the source and the lack of high-sensitivity midinfrared (MIR) detectors. ${ }^{3,9}$ This implies severe practical complications and limitations.

First, when studying living cells or (complex) biofluids, sophisticated cell holders and/or microfluidic systems have to be utilized, with small flow cross sections, complicating sample handling and increasing the danger of clogging. ${ }^{10}$ Second, strong water absorption prevents the investigation of thick samples in transmission, such as large biological cells, cell

Received: December 20, 2019

Accepted: April 30, 2020

Published: April 30, 2020 
complexes (e.g., 3D cell cultures), and (aqueous) tissue. To circumvent the strong water absorption, attenuated total reflection techniques ${ }^{11}$ are often applied, albeit at the cost of even smaller penetration depths. Alternatively, the sample can be dried, which excludes the study of live organisms and strongly alters the sample. ${ }^{12}$

In order to work with larger path lengths and to mitigate the loss in signal-to-noise ratio (SNR) due to strong water absorption, it is necessary to use high-brightness sources like synchrotrons or quantum cascade lasers (QCL). By utilizing their increased MIR power, determination of glucose, lactate, and triglycerides in blood serum with path lengths above 100 $\mu \mathrm{m}\left(1030-1230 \mathrm{~cm}^{-1}\right)^{5}$ and spectroscopy of proteins in aqueous solution in the Amide I and II region with path lengths as large as $32 \mu \mathrm{m}^{13}$ have been demonstrated. However, power scaling is limited, because the strong water absorption eventually results in heating of the samples. Therefore, a further increase of the path length and/or sensitivity is not readily achievable when the remaining transmitted intensity is directly measured with (noisy) MIR photodetectors.

In this work, we demonstrate in theory and experiment the potential of field-resolved infrared spectroscopy (FRS) ${ }^{14}$ to overcome these long-standing limitations. FRS relies on the excitation of resonant molecular vibrations with waveformstable, broadband MIR pulses and electric-field-resolved detection of the emerging fingerprint waveforms. This brings about two major advantages. First, the measurement signal scales linearly with the electric field and, therefore, it decreases with the square root of intensity attenuation. This effectively squares the measurement intensity dynamic range, which is usually limited by the detector dynamic range. ${ }^{15-17}$ Second, temporal isolation of the resonant molecular signal from the impulsive excitation renders FRS robust against fluctuations of the latter. $^{14}$

Together, these properties result in linear scaling of the measurement SNR with the electric field. In the following, we refer to this scaling behavior as field-scaling in order to distinguish it from intensity-scaling techniques whose SNR scales linearly with the light intensity reaching the detector (e.g., conventional FTIR spectrometry). Although field-scaling is not unique to FRS, with other methods, the presence of the time-integrated sample signal (containing all noise contribution from the source) on the detector(s) makes it much more challenging to achieve this regime, especially in conjunction with high-power MIR sources. When applicable, field-scaling of the SNR brings about fundamental advantages for measurements of strongly absorbing samples.

For each SNR-scaling regime, we derive scaling laws for the limit of detection (LOD) of a given analyte in a matrix material (e.g., liquid water) having the absorption coefficient $\alpha_{\mathrm{M}}$ and considering the total interaction length $x$. We show that for field-scaling-in comparison to intensity-scaling-a lower LOD can be obtained for a significantly wider range of interaction lengths, which effectively increases the useable range of path lengths in applications (Figure 1). We find that the theoretical optimum sample thickness is twice the length considered optimum for FTIR measurements. ${ }^{18,19}$ Finally, we experimentally validate this formula, demonstrating that fieldscaling and intensity-noise robust detection can be achieved in practice.

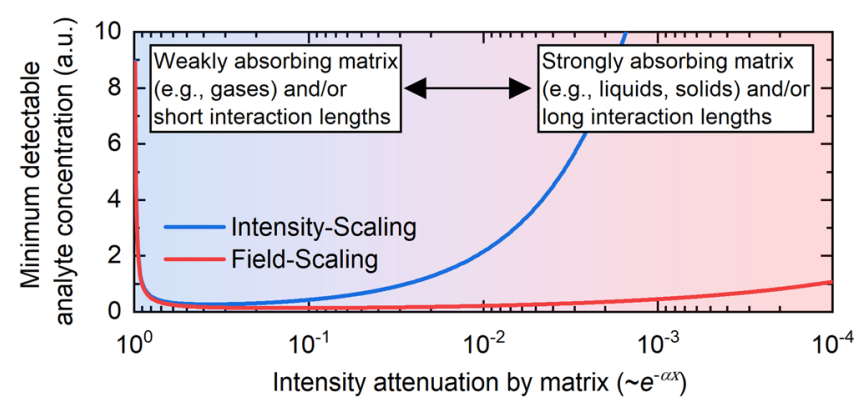

Figure 1. Theoretical limit of detection (LOD) of a given analyte embedded within a matrix substance for field- and intensity-scaling techniques. The theoretical LOD values were calculated assuming a spectrometry device for both approaches offering the same SNR for a non-attenuated beam. For weak attenuations by the matrix, the LOD of field-scaling and intensity-scaling techniques are comparable. For strong attenuations, field-scaling is advantageous.

\section{SCALING LAWS AND OPTIMUM ITERACTION LENGTH}

For a spectroscopic measurement, the optimum path length depends on the SNR-scaling with intensity I. Typically, a distinction into one of the three following scaling-regimes is possible: ${ }^{2,20}$

(1) Square-root-scaling $(\mathrm{SNR} \sim \sqrt{I})$

(2) Linear scaling $(\mathrm{SNR} \sim I)$

(3) Constant (SNR const)

The majority of MIR spectroscopies operate either in the second or the third regime. For a given setup, the "operating regime" often depends on the average signal power reaching the detector. ${ }^{20}$ Usually, a system is intensity-noise limited at high optical powers and, for decreasing power, eventually becomes detector-noise limited. This has to be considered when choosing the interaction length. By contrast, FRS has been demonstrated to afford field-scaling operation for a range of powers extending from a few hundred photons per second to tens of milliwatts ${ }^{14}$ and, potentially, to Watt-level average powers. $^{21}$

For all scaling regimes, derivations of the optimum sample thickness can be found in the Supporting Information. In the following, we focus on the discussion of scaling laws for FRS (i.e., field-scaling). Subsequently, we discuss to what extent these apply to other spectroscopic techniques and how they compare to those of conventional FTIR spectroscopy.

For any spectroscopic measurement, it is desirable to record the molecular signal with the highest possible SNR. A general approach to gaining a stronger signal is to increase the interaction length with the analyte under test. However, this brings about an attenuation of the light intensity due to interaction with the matrix containing the analyte and, for large path lengths, eventually decreases the (relative) measurement SNR. This is particularly severe for aqueous solutions, as the strong IR absorption of water is, unlike the molecules dissolved in it, mostly of little spectroscopic interest. Therefore, it is desirable to optimize the interaction length with the sample under this condition.

According to Beer's absoprtion law, the light intensity I (at a certain frequency) after passing through a medium with absorption coefficient $\alpha$ and propagation length $x$ is given by $I$ $=I_{0} \mathrm{e}^{-\alpha x}$, where $I_{0}$ denotes the intensity before the medium. For the case of a field-scaling time-domain measurement, we 
have previously derived a simple formula for the minimum value of $(\alpha x)_{\text {min }}$ detectable for a particular absorption line of a molecule: ${ }^{14}$

$$
(\alpha x)_{\min }=\frac{2}{\mathrm{DR}_{\mathrm{E}}} \exp \left(\frac{t_{\mathrm{B}}}{T_{\mathrm{L}}}\right)
$$

Here, the electric-field dynamic range $D R_{E}$ is defined as the ratio of the spectral amplitude of the electric field to the detection noise for a given instrument setting (total measurement time, etc.); $T_{\mathrm{L}}$ denotes the dephasing time of the considered absorption line. For a Lorentzian-shaped absorption with a spectral width $v$, the decay time is $T_{\mathrm{L}}=(\pi c v)^{-1}$, with $c$ being the speed of light. The parameter $t_{\mathrm{B}}$ is defined as the instant when the temporal window for an infraredbackground-free measurement begins. ${ }^{14}$

Now, let $\alpha_{\mathrm{M}}$ denote the absorption of water (or any other matrix substance) and $\alpha_{\mathrm{A}}$, the absorption of analyte molecules. By increasing the interaction length $x$, the MIR beam is mainly attenuated due to water absorption according to Beer's law. The electric field dynamic range for a certain optical frequency $v$ scales as $\mathrm{DR}_{\mathrm{E}}(\nu)=\mathrm{DR}_{\mathrm{E}}{ }^{0} \times \exp \left(-\frac{\alpha_{\mathrm{M}}(\nu) x}{2}\right)$ with $\mathrm{DR}_{\mathrm{E}}{ }^{0}$ being the dynamic range for the measurement of the unattenuated radiation. Equation 1 now writes:

$$
\alpha_{\mathrm{A}} x=\frac{2}{\mathrm{DR}_{\mathrm{E}} \mathrm{e}^{0\left(-\alpha_{\mathrm{M}} x\right) / 2}} \exp \left(\frac{t_{\mathrm{B}}}{T_{\mathrm{L}}}\right)
$$

The minimum for $\alpha_{\mathrm{A}}$ is reached for (see the Supporting Information):

$$
x=x_{\mathrm{opt}}^{F}=2 / \alpha_{\mathrm{M}}
$$

According to eq 3, the optimum interaction length is independent of the system's dynamic range and source intensity noise. Therefore, for any FRS system, the choice of an optimum liquid cuvette will depend not on the particular specifications of the system but on only the absorption coefficient $\alpha_{\mathrm{M}}$ of the matrix substance at the desired wavelength.

For broadband spectroscopy, the choice of interaction length becomes more complicated as $\alpha_{\mathrm{M}}$ might vary within the spectral range of interest. This is for instance the case for aqueous samples, for which MIR spectroscopy is most informative between 1000 and $3000 \mathrm{~cm}^{-1}$. Due to the strong water absorption band at $1640 \mathrm{~cm}^{-1}(\mathrm{HOH}$ bending vibration), the optimum interaction length for FRS is 7.4 $\mu \mathrm{m}$, while at $2630 \mathrm{~cm}^{-1}$, it is $178 \mu \mathrm{m}$ (Figure 2). In order to maintain good measurement performance over the entire spectral range, we suggest choosing a path length of $\sim 25 \mu \mathrm{m}$. This keeps the expected LOD within $33 \%$ of the optimum value at all wavenumbers (see the Supporting Information).

It is noteworthy that similar scaling laws apply to any spectroscopic method whose SNR scales linearly with the field strength. For example, Withayachumnankul et al. ${ }^{22}$ obtained a similar expression for the optimum path length in $\mathrm{THz}$ timedomain spectroscopy. ${ }^{23}$ However, their derivation assumes that multiplicative noise (i.e., relative intensity noise) from the source is negligible, without discussing under what circumstances this condition is met.

Similar considerations can be applied to asymmetric FTIR spectrometers where the sample is placed in one arm of the interferometer. ${ }^{20}$ However, due to limited dynamic range and
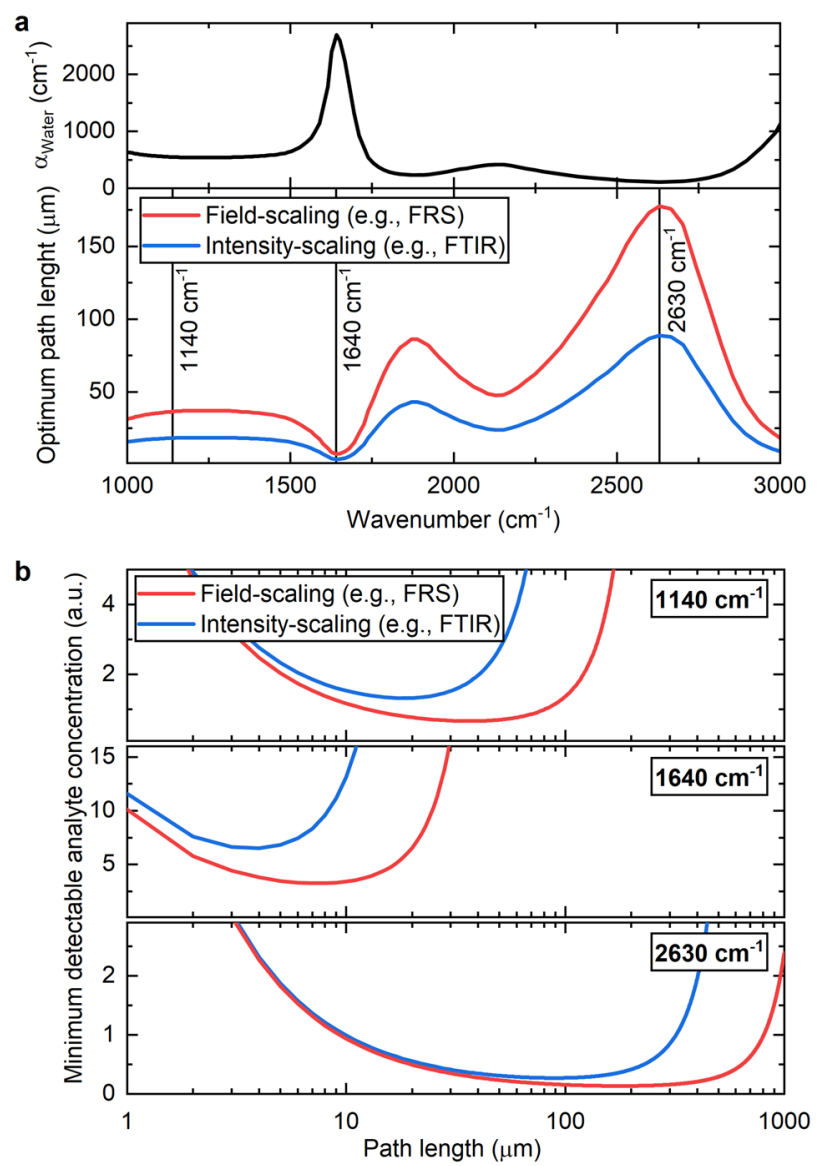

Figure 2. (a) Optimum sample thickness for measurements in aqueous media in dependence of the wavenumber for field-scaling (blue) and intensity-scaling (red) techniques. The absorption coefficient of water (top panel) determines the path length for optimum sensitivity. (b) Theoretical limit of detection (LOD) of a given analyte dissolved in water in dependence of the thickness of the measurement cuvette at the different wavenumbers.

poor noise performance of the available MIR detectors as well as due to source intensity noise, the favorable scaling behavior with intensity attenuation may either not be reached at all or only within a smaller range.

The discussed scaling laws and optimum interaction lengths are in sharp contrast to spectroscopic methods whose SNR is linearly proportional to the light intensity, as is the case for conventional (i.e., symmetric) FTIR spectrometers. ${ }^{2}$ For the comparison of the two scaling regimes, in the following, we consider field- and intensity-scaling devices (e.g., a symmetric and an asymmetric FTIR) with the same performance in terms of minimum detectable absorption difference for nonattenuated beams.

For weak attenuation (i.e., short path length and/or weakly absorbing matrix), the LOD is comparable (Figures 1 and $2 b$ ). For strong attenuation by the matrix (i.e., long path length and/or strongly absorbing matrix), field-scaling is clearly advantageous. This becomes particularly important for samples with nonuniform thickness (e.g., biological tissue or living cells) or varying total absorption and/or water content. In addition, the field-scaling device inherently reaches a twice as low LOD at an optimum path length that is twice the one of the intensity-scaling devices (Figure $2 \mathrm{a}$ and the Supporting Information). 
In principle, SNR-scaling with field strength can also be obtained with direct absorption spectroscopy (DAS) in the MIR range. However, in practice, for DAS, this is only applicable when MIR shot-noise-limited performance can be reached over the entire measurement dynamic range. This is technologically very challenging due to typically orders of magnitude stronger source intensity noise. Additionally, shotnoise-limited performance can only be achieved for high MIR powers, as the noise-equivalent shot-noise power has to overcome the noise of the MIR photodetectors. When Brandstetter et al. ${ }^{5}$ investigated the path length with maximum measurement SNR for a QCL-based DAS setup, they found it to be $140 \mu \mathrm{m}$, determined by the intensity noise of the source. In general, when a spectroscopic system is intensity-noise limited, the full potential of the light source (set by photon shot noise) cannot be exploited and the SNR-maximized path length in experiment will be longer than the optimum value in a detection-limited setting (see the Supporting Information).

\section{EXPERIMENTAL SETUP}

In the remainder of the paper, we verify these theoretical considerations experimentally, employing the mid-infrared field-resolving spectrometer described in detail in our previous work $^{14}$ (see Figure 3). In short, a Kerr-lens mode-locked

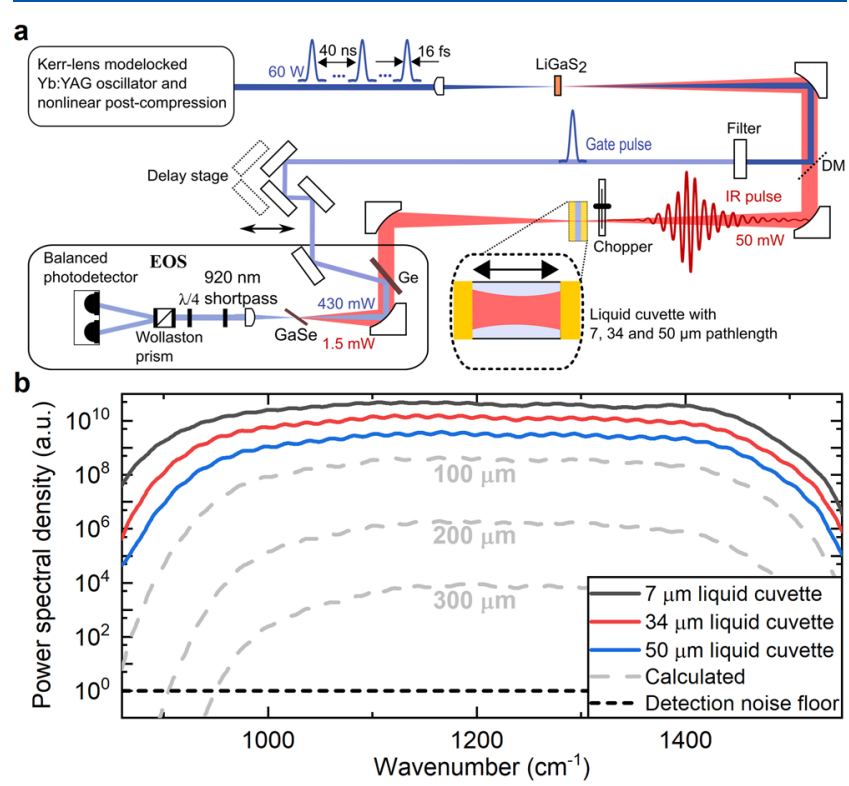

Figure 3. (a) Layout of the field-resolved spectrometer. For this experiment, measurement cuvettes with 7, 34, and $50 \mu \mathrm{m}$ path lengths were used. (b) Intensity dynamic range of the FRS spectrometer after passing through water layers of 7,34 , and $50 \mu \mathrm{m}$ thicknesses, measured over $20 \mathrm{~s}$, with a spectral resolution of $10 \mathrm{~cm}^{-1}$. The gray dashed lines show the expected dynamic range after transmission through 100,200 , and $300 \mu \mathrm{m}$ of water, respectively.

Yb:YAG thin-disc oscillator emits a $28 \mathrm{MHz}$ repetition-rate train of $220 \mathrm{fs}$ pulses spectrally centered at $1030 \mathrm{~nm}$, with an average power of $100 \mathrm{~W}$. The near-infrared (NIR) pulses are spectrally broadened in three nonlinear multipass bulktransmission stages ${ }^{24}$ and temporally compressed to $16 \mathrm{fs}^{25}$ Few-cycle mid-infrared pulses, spectrally covering the 910$1530 \mathrm{~cm}^{-1}$ range at $-20 \mathrm{~dB}$ spectral intensity with an average power of $50 \mathrm{~mW}$, are obtained via intrapulse difference frequency generation (IPDFG) in a $\mathrm{LiGaS}_{2}$ crystal. After the
IPDFG stage, the NIR pulses are separated from the MIR beam via a custom-designed dichroic mirror, recompressed to $16 \mathrm{fs}$, and optically delayed with respect to the MIR transients via a mechanical stage. After passing through a chopper wheel modulating the signal at $10 \mathrm{kHz}$ for lock-in detection, the MIR radiation traverses a liquid cuvette for transmission measurements. Custom multilayer optics were used to compensate for the dispersion of the MIR pulse upon propagation through the $\mathrm{ZnSe}$ windows of the liquid cuvette and temporally compressed the MIR pulse to $59 \mathrm{fs}$ (intensity envelope full width at half-maximum). ${ }^{26}$

The MIR and NIR beams are temporally and spatially recombined at a germanium plate and sent to an electro-optic sampling detection system ${ }^{14,27,28}$ that allows for recording the MIR waveform. To increase measurement stability, the entire MIR beam path was put under vacuum conditions and an active intensity noise stabilization ${ }^{29}$ as well as interferometric delay tracking ${ }^{30}$ of the interferometer were implemented.

\section{RESULTS}

The power spectrum (Figure $3 \mathrm{~b}$ ) was obtained via Fourier transforming the recorded time trace (Figure 4a). After passing through liquid cuvettes with path lengths of 7,34 , and $50 \mu \mathrm{m}$, we obtained peak intensity dynamic range values of $5 \times 10^{10}, 2$ $\times 10^{10}$, and $0.5 \times 10^{10}$ for a total measurement time of $20 \mathrm{~s}$ with a spectral resolution of $10 \mathrm{~cm}^{-1}$, respectively. The wide intensity dynamic range renders the instrument advantageous for measurements of strongly absorbing (aqueous) samples. This is also illustrated in Figure $3 b$, showing the calculated power spectral density of the spectrum after transmission through 100, 200, and $300 \mu \mathrm{m}$ of water.

In addition to the instrument's ability to detect electric fields with high sensitivity, the time-domain nature of FRS renders the detection of molecular emission robust against fluctuations of the excitation. This advantage is described in detail in our previous work ${ }^{14}$ and will be illustrated here for the example of dimethyl sulfone $\left(\mathrm{DMSO}_{2}\right)$, a test molecule used for the measurements presented in this work. The spectral absorption and phase information on $\mathrm{DMSO}_{2}$ can be obtained from the corresponding reference and sample measurements (Figure $4 a, b)$. However, this procedure becomes unstable for small absorptions due to signal fluctuations in the range of the excitation, affecting the (complex) Fourier-transformed spectra.

Measuring the time-domain response to an ultrashort MIR excitation pulse allows for the temporal separation of the sample response of the molecules under investigation and the- orders of magnitude stronger-excitation energy remaining after transmission through the sample. Consequently, the noise power carried by the excitation can be temporally separated from the molecular signal. For substances in aqueous environments, the resonant molecular response usually spans over a few picoseconds. In the case of $\mathrm{DMSO}_{2}$, the dephasing time of the absorption line at $1139 \mathrm{~cm}^{-1}$ is $\sim 0.8 \mathrm{ps}$, which is significantly longer than the full-width at half-maximum of 59 fs of the excitation pulse (Figure 4a). Already $500 \mathrm{fs}$ after the pulse, the signal from the reference pulse drops to $\sim 0.5 \%$ of the peak value. Within this strongly reduced background, a significantly weaker signal of the excited molecules can be detected, as the detrimental influence of the excitation fluctuations is strongly reduced.

This becomes apparent when extracting the resonant molecular response by subtracting a reference from a sample 

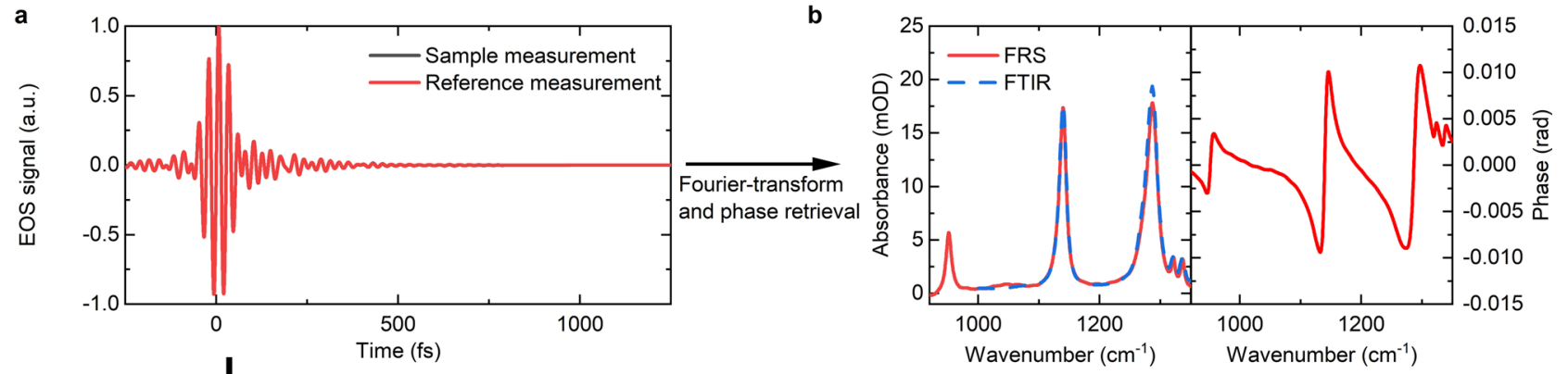

Numerical difference

between sample and reference trace
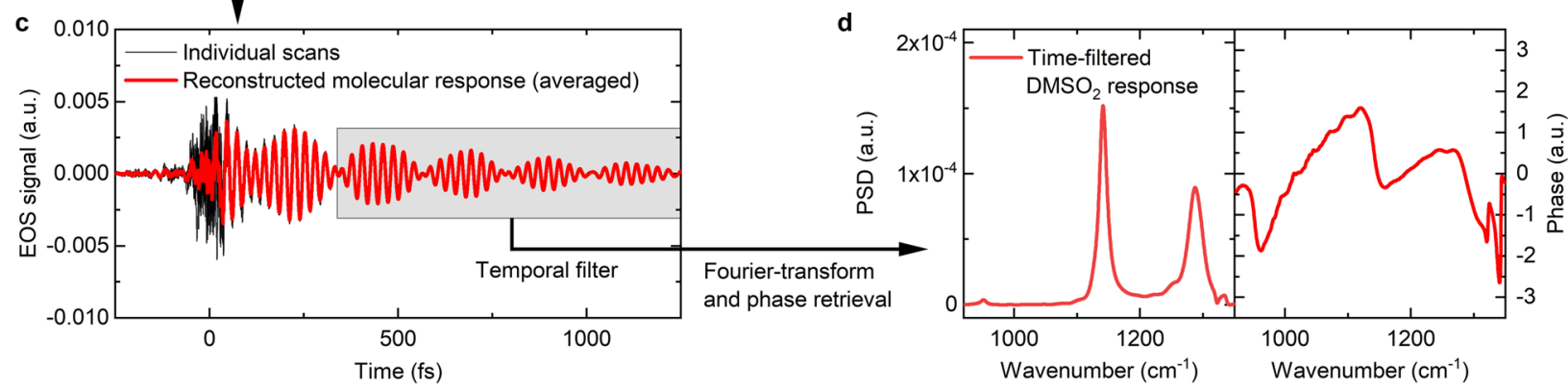

Figure 4. FRS measurement of a solution of $\mathrm{DMSO}_{2}$ in water. (a) EOS traces of a reference and a test sample (liquid cuvette filled with pure water and one with a $1 \mathrm{mg} / \mathrm{mL}$ solution of $\mathrm{DMSO}_{2}$ in water, respectively). The difference between the two traces is miniscule (cannot be discerned by eye). (b) Amplitude and phase spectra obtained via Fourier transform (FT) of the traces in (a), permitting the calculation of conventional absorption and phase spectra of the analyte $\mathrm{DMSO}_{2}$ in its aqueous environment. For comparison, an absorption spectrum of the same solution, measured with a commercial FTIR spectrometer device, is shown. (c) Time-domain molecular response of $\mathrm{DMSO}_{2}$ was obtained via numerical subtraction of the sample from the reference trace, as shown in (a). Due to fluctuations in the excitation remaining after transmission through the sample (induced by intensity and phase noise of the source, by interferometer fluctuations etc.), the reconstruction of the molecular response is noisy in time windows with large EOS signal-in particular during the excitation pulse centered around 0 fs. After several hundred femtoseconds, this detrimental influence of source noise on the subtraction result becomes negligible because of the rapid decay of the excitation pulse. This opens up the window for background-free measurements of the molecular signal. (d) Similar to the conventional spectra shown in (b), one can obtain analyte-specific amplitude and phase spectra of the time-filtered sample response via FT of the truncated time-domain difference trace.

measurement (Figure 4c). During the excitation, source intenstiy fluctuations hinder the clean subtraction, i.e., referencing, of two strong signals, leading to noise in the signal of interest in this time window. After a few hundred femtoseconds, the excitation pulse has decayed either below the detection noise floor or it has become weak enough to be subtracted (or referenced out). Note that a signal of the excited matrix molecules might be spanning over several picoseconds and therefore potentially masking the molecular signal of the analyte. However, vibrations of liquid water dephase within tens of femtoseconds and therefore do not affect the detection of the resonant analyte response in the wake of the excitation. In practice, this means that after a certain time $t_{\mathrm{B}}$, the detection of the molecular signal is only limited by the detection noise and not by any technical noise of the driving laser or of the interferometer. ${ }^{14}$

In our case, the window for background-free access to resonant analyte response opens up approximately $500 \mathrm{fs}$ after the peak of the excitation pulse. Fourier-transforming the timefiltered molecular signal delivers a sample-specific "resonant fingerprint", i.e., a power and a phase spectrum (Figure 4d). Even though its appearance differs from that of conventional spectra, the main spectral features are contained and can be used for quantitative identification of the analyte.

In order to validate eq 2, we evaluated the LOD for $\mathrm{DMSO}_{2}$ dissolved in water in a dilution series, using liquid cuvettes with thicknesses of 7 (commonly used in FTIR spectrometers), 34, and $50 \mu \mathrm{m}$. To retrieve the concentration of $\mathrm{DMSO}_{2}$ for each sample, a calibration measurement at a concentration of $1 \mathrm{mg} /$ $\mathrm{mL}$ was performed for each thickness and used for 1-parameter fits yielding the concentrations of the individual samples. ${ }^{14}$

For a spectral resolution of $4 \mathrm{~cm}^{-1}$ (corresponding to an 8.3 ps time window) and a total effective measurement time of 90 $\mathrm{s}$ per injected sample (45 s each for reference and sample measurement), we experimentally obtained LOD values of 1.6, 0.6 , and $0.7 \mu \mathrm{g} / \mathrm{mL}$ for increasing cuvette thickness, respectively (Figure 5). The theoretical LOD curve was calculated using the absorption coefficient of $\mathrm{DMSO}_{2}$ of 12.92 $\mathrm{cm}^{-1}$ for $1 \mathrm{mg} / \mathrm{mL}$ at $1139 \mathrm{~cm}^{-1}$, a decay time $T_{\mathrm{L}}$ of $770 \mathrm{fs}$, the absorption coefficient of water of $537 \mathrm{~cm}^{-1,31}$ and a field

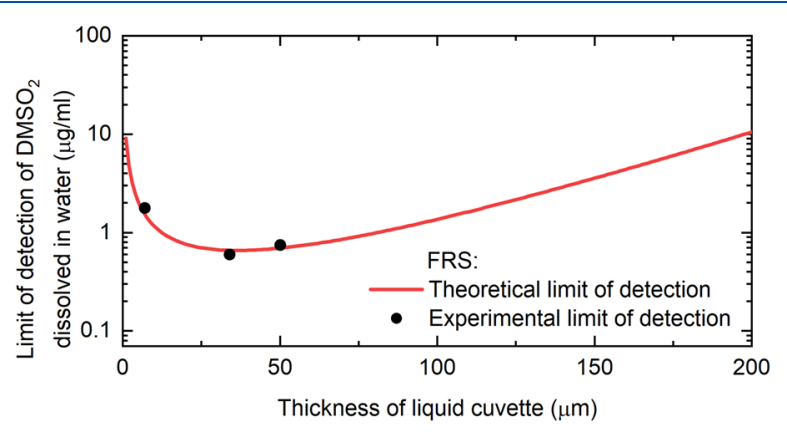

Figure 5. Theoretical vs experimental limits of detection (LOD) for $\mathrm{DMSO}_{2}$ dissolved in water in dependence of the thickness of the measurement cuvette. 
dynamic range $\mathrm{DR}_{\mathrm{E}}{ }^{0}$ of the non-attenuated beam of $3.0 \times 10^{5}$. This theoretical curve is in very good agreement with the experimentally obtained values for the LOD.

\section{CONCLUSION AND OUTLOOK}

We have demonstrated that broadband field-resolved MIR spectroscopy can offer an intensity dynamic range in excess of $10^{10}$ over more than $460 \mathrm{~cm}^{-1}$ (without measurement cuvette) for a measurement time of less than $1 \mathrm{~min}$, rendering it applicable for the investigation of strongly absorbing (aqueous) samples. The ability of FRS to measure the temporally retarded sample response separated from the nonresonant response (i.e., excitation remaining after transmission through the sample) grants access to the-orders of magnitude smaller-molecular signal in a background-reduced manner, rendering the measurement SNR virtually independent from excitation fluctuations. This enables detection sensitivities in the sub-microgram/milliliter range for molecules solved in water. Furthermore, the signal strength and SNR scale linearly with the electric field of the excitation source. Based on these properties, we theoretically derived and experimentally confirmed that in FRS the optimum interaction length with a sample, $x_{\mathrm{opt}}=2 / \alpha_{\mathrm{M}}$, only depends on the absorption coefficient $\alpha_{\mathrm{M}}$ of the matrix substance.

For many biological samples, the matrix substance is water, therefore, we further analyzed this case and evaluated the measurement performance in dependence of wavelength and thickness. We found that sub-microgram/milliliter detection sensitivities can be maintained for samples as thick as $80 \mu \mathrm{m}$ and that, even for $0.2 \mathrm{~mm}$ thick samples, LOD values in the range of $10 \mu \mathrm{g} / \mathrm{mL}$ are feasible, which is the level achieved by state-of-the-art research-grade FTIR instruments, ${ }^{14}$ albeit under the stringent condition of sub-10 $\mu \mathrm{m}$ sample thickness. In the fingerprint region from 1000 to $3000 \mathrm{~cm}^{-1}$, the measurement performance can be kept within $33 \%$ of the peak performance at all wavenumbers by choosing a sample thickness around $25 \mu \mathrm{m}$. Rapid advances of femtosecond technology hold promise for the extension of FRS to the coverage of the entire molecular fingerprint region in the near future. $^{32,33}$ At the same time, further improvement of the sensitivity of electro-optic sampling will push the limit of detection to the low nanogram/milliliter range or below. ${ }^{34}$

Compared to conventional (i.e., symmetric) FTIR spectrometers, the scaling laws of FRS for the sensitivity in dependence of the sample thickness and total absorption are highly advantageous. The relaxed requirements to the sample thickness enable a more flexible design of liquid cuvettes and microfluidic chips for MIR spectroscopic applications. In addition, the increased SNR for the measurement of thick aqueous samples will be beneficial for MIR transmission spectroscopy and spectroscopy-microscopy of biological samples, such as living cells, bulk cells, and tissue cultures as well as biological tissues, in their natural (hydrated) state.

\section{ASSOCIATED CONTENT}

\section{s) Supporting Information}

The Supporting Information is available free of charge at https://pubs.acs.org/doi/10.1021/acs.analchem.9b05744.

Discussions of scaling laws for the limit of detection in aqueous media for intensity- and field-scaling spectroscopic methods and relative-intensity noise dominated measurements (PDF)

\section{AUTHOR INFORMATION}

\section{Corresponding Authors}

Marinus Huber - Max Planck Institute of Quantum Optics, Garching 85748, Germany; Ludwig Maximilians University München, Garching 85748, Germany; 이이이.org/00000001-5309-4475; Email: marinus.huber@mpq.mpg.de

Ioachim Pupeza - Max Planck Institute of Quantum Optics, Garching 85748, Germany; Ludwig Maximilians University München, Garching 85748, Germany; ㅇo이.org/00000001-8422-667X; Email: ioachim.pupeza@mpq.mpg.de

\section{Authors}

Michael Trubetskov - Max Planck Institute of Quantum Optics, Garching 85748, Germany

Syed A. Hussain - Max Planck Institute of Quantum Optics, Garching 85748, Germany; Ludwig Maximilians University München, Garching 85748, Germany

Wolfgang Schweinberger - Max Planck Institute of Quantum Optics, Garching 85748, Germany; Department of Physics and Astronomy, King Saud University, Riyadh 11451, Saudi Arabia

Christina Hofer - Max Planck Institute of Quantum Optics, Garching 85748, Germany; Ludwig Maximilians University München, Garching 85748, Germany

Complete contact information is available at:

https://pubs.acs.org/10.1021/acs.analchem.9b05744

\section{Notes}

The authors declare no competing financial interest.

\section{REFERENCES}

(1) Diem, M.; Griffiths, P. R.; Chalmers, J. M. Vibrational Spectroscopy for Medical Diagnosis; Wiley: Chichester, 2008; Vol. 40.

(2) Griffiths, P. R.; De Haseth, J. A. Fourier Transform Infrared Spectrometry; John Wiley \& Sons, 2007; Vol. 171.

(3) Yang, H.; Yang, S.; Kong, J.; Dong, A.; Yu, S. Nat. Protoc. 2015, 10 (3), 382-396.

(4) Martin, F. L.; Kelly, J. G.; Llabjani, V.; Martin-Hirsch, P. L.; Patel, I. I.; Trevisan, J.; Fullwood, N. J.; Walsh, M. J. Nat. Protoc. 2010, 5 (11), 1748-1760.

(5) Brandstetter, M.; Volgger, L.; Genner, A.; Jungbauer, C.; Lendl, B. Appl. Phys. B: Lasers Opt. 2013, 110 (2), 233-239.

(6) Movasaghi, Z.; Rehman, S.; Ur Rehman, D. I. Appl. Spectrosc. Rev. 2008, 43 (2), 134-179.

(7) Ollesch, J.; Theegarten, D.; Altmayer, M.; Darwiche, K.; Hager, T.; Stamatis, G.; Gerwert, K. Biomed. Spectrosc. Imaging 2016, 5 (2), 129-144.

(8) Hands, J. R.; Clemens, G.; Stables, R.; Ashton, K.; Brodbelt, A.; Davis, C.; Dawson, T. P.; Jenkinson, M. D.; Lea, R. W.; Walker, C.; Baker, M. J. J. Neuro-Oncol. 2016, 127 (3), 463-472.

(9) Rogalski, A. Infrared Detectors; CRC press, 2010.

(10) Vaccari, L.; Birarda, G.; Businaro, L.; Pacor, S.; Grenci, G. Anal. Chem. 2012, 84 (11), 4768-4775.

(11) Kazarian, S. G.; Chan, K. L. A. Analyst 2013, 138 (7), 1940.

(12) Cameron, J. M.; Butler, H. J.; Palmer, D. S.; Baker, M. J. J. Biophotonics 2018, 11 (4), No. e201700299.

(13) Schwaighofer, A.; Montemurro, M.; Freitag, S.; Kristament, C.; Culzoni, M. J.; Lendl, B. Anal. Chem. 2018, 90 (11), 7072-7079.

(14) Pupeza, I.; Huber, M.; Trubetskov, M.; Schweinberger, W.; Hussain, S. A.; Hofer, C.; Fritsch, K.; Poetzlberger, M.; Vamos, L.; Fill, E.; Amotchkina, T.; Kepesidis, K. V.; Apolonski, A.; Karpowicz, N.; Pervak, V.; Pronin, O.; Fleischmann, F.; Azzeer, A.; Žigman, M.; Krausz, F. Nature 2020, 577 (7788), 52-59.

(15) Snyder, J. J. Appl. Opt. 1988, 27 (21), 4465.

(16) Jarry, G.; Poupinet, L.; Watson, J.; Lepine, T. Appl. Opt. 1995, 34 (12), 2045 
(17) Liukaityte, S.; Lequime, M.; Zerrad, M.; Begou, T.; Amra, C. Opt. Lett. 2015, 40 (14), 3225.

(18) Jensen, P. S.; Bak, J. Appl. Spectrosc. 2002, 56 (12), 1600-1606.

(19) Mark, H. L.; Griffiths, P. R. Appl. Spectrosc. 2002, 56 (5), 633639.

(20) Newbury, N. R.; Coddington, I.; Swann, W. Opt. Express 2010, 18 (8), 7929.

(21) Butler, T. P.; Gerz, D.; Hofer, C.; Xu, J.; Gaida, C.; Heuermann, T.; Gebhardt, M.; Vamos, L.; Schweinberger, W.; Gessner, J. A.; Siefke, T.; Heusinger, M.; Zeitner, U.; Apolonski, A.; Karpowicz, N.; Limpert, J.; Krausz, F.; Pupeza, I. Opt. Lett. 2019, 44 (7), 1730.

(22) Withayachumnankul, W.; Fischer, B. M.; Abbott, D. Opt. Express 2008, 16 (10), 7382.

(23) Jepsen, P. U.; Cooke, D. G.; Koch, M. Laser Photon. Rev. 2011, 5 (1), 124-166.

(24) Schulte, J.; Sartorius, T.; Weitenberg, J.; Vernaleken, A.; Russbueldt, P. Opt. Lett. 2016, 41 (19), 4511.

(25) Fritsch, K.; Poetzlberger, M.; Pervak, V.; Brons, J.; Pronin, O. Opt. Lett. 2018, 43 (19), 4643.

(26) Amotchkina, T.; Trubetskov, M.; Hussain, S. A.; Hahner, D.; Gerz, D.; Huber, M.; Schweinberger, W.; Pupeza, I.; Krausz, F.; Pervak, V. Opt. Lett. 2019, 44 (21), 5210.

(27) Gallot, G.; Grischkowsky, D. J. Opt. Soc. Am. B 1999, 16 (8), 1204.

(28) Wu, Q.; Zhang, X. Appl. Phys. Lett. 1995, 67 (24), 3523-3525.

(29) Huber, M.; Schweinberger, W.; Stutzki, F.; Limpert, J.; Pupeza, I.; Pronin, O. Opt. Express 2017, 25 (19), 22499.

(30) Schweinberger, W.; Vamos, L.; Xu, J.; Hussain, S. A.; Baune, C.; Rode, S.; Pupeza, I. Opt. Express 2019, 27 (4), 4789.

(31) Segelstein, D. J. The Complex Refractive Index of Water. University of Missouri: Kansas City, 1981.

(32) Butler, T. P.; Lilienfein, N.; Xu, J.; Nagl, N.; Hofer, C.; Gerz, D.; Mak, K. F.; Gaida, C.; Heuermann, T.; Gebhardt, M.; Limpert, J.; Krausz, F.; Pupeza, I. J. Phys. Photonics 2019, 1 (4), 044006.

(33) Wang, Q.; Zhang, J.; Kessel, A.; Nagl, N.; Pervak, V.; Pronin, O.; Mak, K. F. Opt. Lett. 2019, 44 (10), 2566.

(34) Hofer, C.; Hussain, S. A.; Schweinberger, W.; Huber, M.; Butler, T. P.; Gerz, D.; Karpowicz, N.; Krausz, F.; Pupeza, I. Quantum-Efficiency and Bandwidth Optimized Electro-Optic Sampling. 2019 Conference on Lasers and Electro-Optics Europe \& European Quantum Electronics Conference (CLEO/Europe-EQEC); IEEE, 2019; $\mathrm{p}$ 1 . . 